\title{
APPENDIX 10
}

\section{New Biomass Equations}

i-Tree tree biomass allometric equations are found in appendix 6 (see also Nowak 1994c and Nowak et al. 2002b). Various biomass equations in Table 27 will be added to i-Tree in 2020 from GlobAllomeTree (2017) to improve carbon estimates. These equations estimate either aboveground dry weight biomass or total tree dry weight biomass.

Table 27.-New biomass equations and variables, by species, from GlobAllomeTree 2017. Where multiple equations exist, the average results from all equations are used. $A G B=$ aboveground dry weight biomass; $T B=$ total tree dry weight biomass; $x=$ d.b.h.

\begin{tabular}{|c|c|c|c|c|c|c|}
\hline Species & Equation form & B & B1 & B2 & Units ( $x$ ) & Output (Y) \\
\hline Acacia auriculaeformis & $Y=10^{(B+(B 1 * \log (x)))}$ & -1.29248 & 2.305227 & & $(\mathrm{~cm})$ & AGB (kg) \\
\hline Acacia auriculaeformis & $Y=10^{(B+(B 1 * \log (x)))}$ & -1.57926 & 2.436945 & & $(\mathrm{~cm})$ & $\mathrm{AGB}(\mathrm{kg})$ \\
\hline Acacia auriculaeformis & $Y=10^{(B+(B 1 * \log (x)))}$ & -1.3577 & 2.4177 & & $(\mathrm{~cm})$ & TB $(\mathrm{kg})$ \\
\hline Acacia nilotica & $Y=B+B 1 * x^{2}$ & -21.4868 & 0.5797 & & $(\mathrm{~cm})$ & $\mathrm{AGB}(\mathrm{kg})$ \\
\hline Acer rubrum & $Y=B^{*}\left(\left(x^{2}\right)^{B 1}\right)$ & 2.62288 & 1.21132 & & $\left(\mathrm{in}^{2}\right)$ & AGB (lb) \\
\hline Acer saccharum & $Y=10^{(B+(B 1 * \log (x)))}$ & 2.2151 & 2.4209 & & $(\mathrm{~cm})$ & $\mathrm{TB}(\mathrm{g})$ \\
\hline Adinandra glischrolomavar & $Y=B^{*} x^{B 1}$ & 0.1142 & 2.4451 & & $(\mathrm{~cm})$ & AGB (kg) \\
\hline Alnus sp. & $Y=\left(B^{*} x^{2}\right)-B 1$ & 0.2896 & 5.5963 & & $\left(\mathrm{~cm}^{2}\right)$ & AGB (kg) \\
\hline Alogus nepalensis & $Y=B^{*} x^{B 1}$ & 1.089 & 1.6481 & & $(\mathrm{~cm})$ & AGB $(\mathrm{kg})$ \\
\hline Alogus nepalensis & $Y=B^{*} x^{B 1}$ & 1.5776 & 1.6071 & & $(\mathrm{~cm})$ & TB $(\mathrm{kg})$ \\
\hline Artocarpus lakoocha & $Y=B^{*} x^{B 1}$ & 0.1245 & 2.4163 & & $(\mathrm{~cm})$ & AGB (kg) \\
\hline Avicennia germinans & $Y=10^{B *} x^{B 1}$ & -0.395 & 1.934 & & $(\mathrm{~cm})$ & AGB (kg) \\
\hline Bambusa balcooa & $Y=10^{\left(B+\left(B 1^{*} \log (x)\right)\right)}$ & 2.149 & 2.284 & & $(\mathrm{~cm})$ & AGB (g) \\
\hline Bambusa balcooa & $\mathrm{Y}=10^{\left(\mathrm{B}+\left(\mathrm{B} 1^{*} \log (\mathrm{x})\right)\right)}$ & 2.368 & 2.214 & & $(\mathrm{~cm})$ & AGB (g) \\
\hline Bambusa balcooa & $\mathrm{Y}=10^{(\mathrm{B}+(\mathrm{B} 1 * \log (\mathrm{x})))}$ & 2.199 & 2.353 & & $(\mathrm{~cm})$ & AGB (g) \\
\hline Bambusa cacharensis & $\mathrm{Y}=10^{(\mathrm{B}+(\mathrm{B} 1 * \log (\mathrm{x})))}$ & 2.134 & 2.268 & & $(\mathrm{~cm})$ & AGB (g) \\
\hline Bambusa cacharensis & $\mathrm{Y}=10^{(\mathrm{B}+(\mathrm{B} 1 * \log (\mathrm{x})))}$ & 2.184 & 2.178 & & $(\mathrm{~cm})$ & $A G B(g)$ \\
\hline Bambusa cacharensis & $\mathrm{Y}=10^{(\mathrm{B}+(\mathrm{B} 1 * \log (\mathrm{x})))}$ & 2.078 & 2.14 & & $(\mathrm{~cm})$ & $\mathrm{AGB}(\mathrm{g})$ \\
\hline Bambusa cacharensis & $\mathrm{Y}=10^{(\mathrm{B}+(\mathrm{B} 1 * \log (\mathrm{x})))}$ & 2.174 & 2.306 & & $(\mathrm{~cm})$ & $A G B(g)$ \\
\hline Bambusa vulgaris & $Y=10^{(B+(B 1 * \log (x)))}$ & 2.281 & 2.149 & & $(\mathrm{~cm})$ & $\mathrm{AGB}(\mathrm{g})$ \\
\hline Bambusa vulgaris & $Y=10^{\left(B+\left(B 1^{*} \log (x)\right)\right)}$ & 2.554 & 1.956 & & $(\mathrm{~cm})$ & AGB (g) \\
\hline Bambusa vulgaris & $Y=10^{(B+(B 1 * \log (x)))}$ & 2.386 & 2.079 & & $(\mathrm{~cm})$ & AGB (g) \\
\hline Bambusa vulgaris & $Y=10^{(B+(B 1 * \log (x)))}$ & 2.548 & 1.97 & & $(\mathrm{~cm})$ & AGB (g) \\
\hline Betula alleghaniensis & $\mathrm{Y}=10^{\left(\mathrm{B}+\left(\mathrm{B} 1^{*} \log (\mathrm{x})\right)\right)}$ & 2.2264 & 2.415 & & $(\mathrm{~cm})$ & $\mathrm{TB}(\mathrm{g})$ \\
\hline Buddleia megalocephala & $Y=\left(B^{*} x^{2}\right)-B 1$ & 0.2696 & 3.067 & & $\left(\mathrm{~cm}^{2}\right)$ & AGB (kg) \\
\hline Carya spp. & $Y=B^{*}\left(\left(x^{2}\right)^{B 1}\right)$ & 2.96561 & 1.19764 & & $\left(\mathrm{in}^{2}\right)$ & AGB (lb) \\
\hline Carya spp. & $Y=B^{*}\left(\left(x^{2}\right)^{B 1}\right)$ & 3.06011 & 1.19138 & & $\left(\mathrm{in}^{2}\right)$ & AGB (lb) \\
\hline
\end{tabular}

Appendix 10 in: Nowak, David J. 2020. Understanding i-Tree: Summary of programs and methods. General Technical Report NRS-200. Madison, WI: U.S. Department of Agriculture, Forest Service, Northern Research Station. https://doi.org/10.2737/NRS-GTR-200-Appendix10 


\begin{tabular}{|c|c|c|c|c|c|c|}
\hline Species & Equation form & B & B1 & B2 & Units (x) & Output (Y) \\
\hline Carya spp. & $Y=B^{*}\left(\left(x^{2}\right)^{B 1}\right)$ & 2.86238 & 1.20634 & & $\left(\mathrm{in}^{2}\right)$ & AGB (lb) \\
\hline Cassia siamea & $\mathrm{Y}=10^{(\mathrm{B}+(\mathrm{B} 1 * \log (\mathrm{x})))}$ & -1.5851 & 2.4855 & & $(\mathrm{~cm})$ & $\mathrm{TB}(\mathrm{kg})$ \\
\hline Cecropia schreberiana & $Y=B^{*} x^{B 1}$ & 0.16502 & 2.3351 & & $(\mathrm{~cm})$ & AGB (kg) \\
\hline Ceiba pentandra & $Y=B^{*} x^{B 1}$ & 0.16502 & 2.3351 & & $(\mathrm{~cm})$ & AGB (kg) \\
\hline Cercocarpus ledifolius & $Y=B^{*} x^{B 1}$ & 0.0104 & 2.7105 & & $(\mathrm{~cm})$ & AGB $(k g)$ \\
\hline Cinnamomum camphora & $\mathrm{Y}=10^{(\mathrm{B}+(\mathrm{B} 1 * \log (\mathrm{x})))}$ & -0.85 & 2.41 & & $(\mathrm{~cm})$ & $\mathrm{AGB}(\mathrm{kg})$ \\
\hline Cornus florida & $Y=B^{*}\left(\left(x^{2}\right)^{B 1}\right)$ & 2.73676 & 1.21095 & & $\left(\mathrm{in}^{2}\right)$ & AGB (lb) \\
\hline Cornus florida & $Y=B^{*}\left(\left(x^{2}\right)^{B 1}\right)$ & 3.08355 & 1.14915 & & $\left(\mathrm{in}^{2}\right)$ & AGB (lb) \\
\hline Cornus florida & $Y=B^{*}\left(\left(x^{2}\right)^{B 1}\right)$ & 3.37156 & 1.10056 & & $\left(\mathrm{in}^{2}\right)$ & AGB (lb) \\
\hline Cupressus macrocarpa & $Y=B-\left(B 1{ }^{*} x\right)+\left(B 2^{*} x^{2}\right)$ & 34.63 & 9.89 & 0.887 & $(\mathrm{~cm})$ & AGB (kg) \\
\hline Cupressus macrocarpa & $Y=B^{*} x^{B 1}$ & 1.089 & 1.6481 & & $(\mathrm{~cm})$ & AGB (kg) \\
\hline Cupressus macrocarpa & $Y=10^{(B+(B 1 * \log (x)))}$ & -0.435 & 2.12 & & $(\mathrm{~cm})$ & AGB (kg) \\
\hline Cupressus macrocarpa & $Y=B^{*} x^{B 1}$ & 1.5776 & 1.6071 & & $(\mathrm{~cm})$ & $\mathrm{TB}(\mathrm{kg})$ \\
\hline Dalbergia sissoo & $Y=B^{*} x^{B 1}$ & 0.667 & 1.832 & & $(\mathrm{~cm})$ & AGB $(\mathrm{kg})$ \\
\hline Dalbergia sissoo & $Y=B^{*} x^{B 1}$ & 0.611 & 1.752 & & $(\mathrm{~cm})$ & AGB $(\mathrm{kg})$ \\
\hline Daniellia thurifera & $Y=B^{*} x^{B 1}$ & 0.16502 & 2.3351 & & $(\mathrm{~cm})$ & $\mathrm{AGB}(\mathrm{kg})$ \\
\hline Eucalyptus brassiana & $Y=10^{((B * \log (x))-B 1)}$ & 2.39602 & 1.3933 & & $(\mathrm{~cm})$ & $\mathrm{TB}(\mathrm{kg})$ \\
\hline Eucalyptus camaldulensis & $Y=10^{((B * \log (x))-B 1)}$ & 2.39602 & 1.3933 & & $(\mathrm{~cm})$ & $\mathrm{TB}(\mathrm{kg})$ \\
\hline Eucalyptus hybrid & $Y=B^{*} x^{B 1}$ & 0.135336 & 2.416484 & & $(\mathrm{~cm})$ & AGB (kg) \\
\hline Eucalyptus tereticornis & $Y=10^{((B * \log (x))-B 1)}$ & 2.39602 & 1.3933 & & $(\mathrm{~cm})$ & TB $(\mathrm{kg})$ \\
\hline Fagus grandifolia & $Y=10^{(B+(B 1 * \log (x)))}$ & 2.2916 & 2.3916 & & $(\mathrm{~cm})$ & TB (g) \\
\hline Hopea odorata & $Y=B^{*} x^{B 1}$ & 0.1277 & 2.3944 & & $(\mathrm{~cm})$ & AGB (kg) \\
\hline Juniperus virginiana & $Y=B^{*} x^{B 1}$ & 0.1632 & 2.2454 & & $(\mathrm{~cm})$ & AGB $(k g)$ \\
\hline Lagerstroemia calyculata & $Y=B^{*} x^{B 1}$ & 0.1277 & 2.3943 & & $(\mathrm{~cm})$ & AGB $(k g)$ \\
\hline Laguncularia racemosa & $Y=10^{B *} x^{B 1}$ & 0.112 & 1.731 & & $(\mathrm{~cm})$ & AGB $(\mathrm{kg})$ \\
\hline Liquidambar styraciflua & $Y=B^{*}\left(\left(x^{2}\right)^{B 1}\right)$ & 1.67422 & 1.17677 & & (in2) & AGB (lb) \\
\hline Liriodendron tulipifera & $Y=B^{*}\left(\left(x^{2}\right)^{B 1}\right)$ & 1.79951 & 1.22938 & & (in2) & AGB (lb) \\
\hline Liriodendron tulipifera & $Y=B^{*}\left(\left(x^{2}\right)^{B 1}\right)$ & 1.76621 & 1.21948 & & (in2) & AGB (lb) \\
\hline Mangifera minitifolia & $Y=B^{*}\left(x^{B 1}\right)$ & 0.14 & 2.31 & & $(\mathrm{~cm})$ & AGB (kg) \\
\hline Nauclea diderrichii & $Y=B^{*}\left(x^{B 1}\right)$ & 0.16502 & 2.3351 & & $(\mathrm{~cm})$ & AGB (kg) \\
\hline Nyssa sylvatica & $Y=B^{*}\left(\left(x^{2}\right)^{B 1}\right)$ & 2.56795 & 1.18685 & & $\left(\mathrm{in}^{2}\right)$ & AGB (lb) \\
\hline Olneya tesota & $Y=B^{*}\left(\left(x^{2}\right)^{B 1}\right)$ & 2.50008 & 1.19431 & & $\left(\mathrm{in}^{2}\right)$ & AGB (lb) \\
\hline Oxydendrum arboreum & $Y=B^{*}\left(\left(x^{2}\right)^{B 1}\right)$ & 2.37722 & 1.21022 & & $\left(\mathrm{in}^{2}\right)$ & AGB (lb) \\
\hline Picea abies & $Y=B-(B 1 * x)+\left(B 2 * x^{2}\right)$ & 19.018 & 4.806 & 0.565 & $(\mathrm{~cm})$ & AGB $(\mathrm{kg})$ \\
\hline Picea rubens & $Y=10^{B *} x^{B 1} / 1,000,000$ & 2.1735 & 2.1936 & & $(\mathrm{~cm})$ & AGB (Mg) \\
\hline Pinus banksiana & $Y=B^{*} X^{B 1}$ & 1.265 & 2.273 & & (in) & $\mathrm{TB}(\mathrm{kg})$ \\
\hline Pinus caribaea & $Y=B^{*} x^{B 1}$ & 0.07035 & 2.56 & & $(\mathrm{~cm})$ & AGB (kg) \\
\hline Pinus sylvestris & $\mathrm{Y}=\mathrm{B}-\left(\mathrm{B} 1^{*} \mathrm{x}\right)+\left(\mathrm{B} 2^{*} \mathrm{x}^{2}\right)$ & 7.041 & 1.279 & 0.201 & $(\mathrm{~cm})$ & AGB (kg) \\
\hline Pinus sylvestris & $\mathrm{Y}=\mathrm{B}-\left(\mathrm{B} 1{ }^{*} \mathrm{x}\right)+\left(\mathrm{B} 2^{*} \mathrm{x}^{2}\right)$ & 18.779 & 4.328 & 0.506 & $(\mathrm{~cm})$ & AGB $(k g)$ \\
\hline Populus deltoides & $Y=10^{(B+(B 1 * \log (x)))}$ & -1.0922 & 2.4875 & & $(\mathrm{~cm})$ & AGB $(k g)$ \\
\hline
\end{tabular}




\begin{tabular}{|c|c|c|c|c|c|c|}
\hline Species & Equation form & B & B1 & B2 & Units ( $\mathrm{x}$ ) & Output (Y) \\
\hline Populus deltoides & $\mathrm{Y}=10^{\left(\mathrm{B}+\left(\mathrm{B} 1^{*} \log (\mathrm{x})\right)\right)}$ & -1.0387 & 2.4967 & & $(\mathrm{~cm})$ & TB (kg) \\
\hline Quercus alba & $\mathrm{Y}=10^{(\mathrm{B}+(\mathrm{B} 1 * \log (\mathrm{x})))}$ & -1.266 & 2.613 & & $(\mathrm{~cm})$ & AGB $(\mathrm{kg})$ \\
\hline Quercus alba & $Y=B^{*}\left(\left(x^{2}\right)^{B 1}\right)$ & 2.54413 & 1.1859 & & $\left(\mathrm{in}^{2}\right)$ & AGB (Ib) \\
\hline Quercus alba & $Y=B^{*}\left(\left(x^{2}\right)^{B 1}\right)$ & 2.49773 & 1.19895 & & $\left(\mathrm{in}^{2}\right)$ & AGB (lb) \\
\hline Quercus alba & $Y=B^{*}\left(\left(x^{2}\right)^{B 1}\right)$ & 2.50124 & 1.19431 & & $\left(\mathrm{in}^{2}\right)$ & AGB (lb) \\
\hline Quercus ilex & $Y=B^{*} x^{B 1}$ & 0.2306 & 2.2791 & & $(\mathrm{~cm})$ & $\mathrm{AGB}(\mathrm{kg})$ \\
\hline Quercusilex & $Y=B^{*} x^{B 1}$ & 0.2313 & 2.2662 & & $(\mathrm{~cm})$ & $\mathrm{AGB}(\mathrm{kg})$ \\
\hline Quercus prinus & $Y=B^{*}\left(\left(x^{2}\right)^{B 1}\right)$ & 2.93819 & 1.19746 & & $\left(\mathrm{in}^{2}\right)$ & AGB (lb) \\
\hline Quercus sp. & $Y=B^{*} x^{2}+B 1$ & 0.6048 & 4.3198 & & $\left(\mathrm{~cm}^{2}\right)$ & $\mathrm{AGB}(\mathrm{kg})$ \\
\hline Quercus stellata & $Y=B^{*}\left(\left(x^{2}\right)^{B 1}\right)$ & 2.23774 & 1.21527 & & $\left(\mathrm{in}^{2}\right)$ & AGB (lb) \\
\hline Quercus velutina & $\mathrm{Y}=10^{(\mathrm{B}+(\mathrm{B} 1 * \log (\mathrm{x})))}$ & 1.00005 & 2.10621 & & (in) & TB (lb) \\
\hline Rhizophora mangle & $Y=10^{B *} x^{B 1}$ & -0.441 & 1.93 & & $(\mathrm{~cm})$ & $\mathrm{AGB}(\mathrm{kg})$ \\
\hline Tectona grandis & $Y=B^{*} x^{B 1}$ & 0.202 & 2.353 & & $(\mathrm{~cm})$ & TB (kg) \\
\hline Terminalia superba & $Y=B^{*} x^{B 1}$ & 0.066 & 2.565 & & $(\mathrm{~cm})$ & TB (kg) \\
\hline Thuja occidentalis & $Y=10^{(B+(B 1 * \log (x)))}$ & 1.3294 & 1.919 & & (in) & TB (lb) \\
\hline Vitellaria paradoxa & $Y=B^{*} x^{B 1}$ & 0.08 & 2.46 & & $(\mathrm{~cm})$ & TB (kg) \\
\hline
\end{tabular}

